\title{
Slow and growing slower
}

Nature 553, 186-188 (2018)

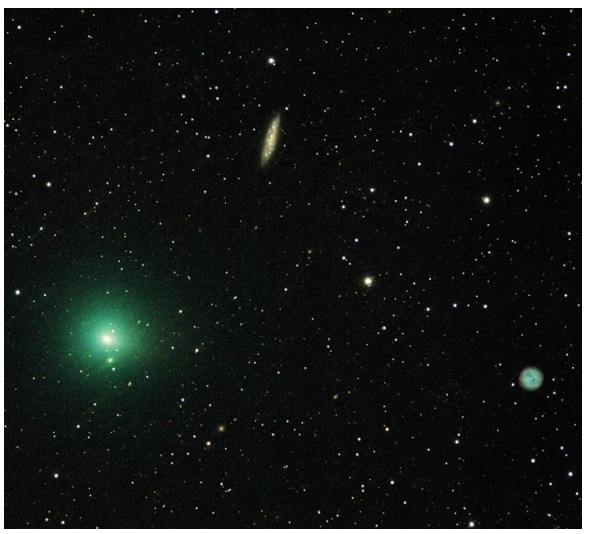

Credit: Kees Scherer

Cyanogen gas is colourless and toxic, but its fluorescence in sunlight makes it an ideal probe of the rotation rate of a comet's nucleus. As the comet spins, sublimated gas moves outwards and traces spiral arms, whose repeating patterns reveal the rotation period. Indeed, Dennis Bodewits and co-authors used the Large Monolithic Imager on the Discovery Channel Telescope at Lowell Observatory to map the cyanogen jets from comet 41P/Tuttle-GiacobiniKresák (41P; pictured in green) in March 2017, inferring a period of 19.75-20.05 h during 5-9 March. Two weeks later, the period had lengthened to almost $27 \mathrm{~h}$.

By 7-9 May, photometric observations using the UltraViolet-Optical Telescope on the Swift satellite showed that the period had rapidly stretched to $46-60 \mathrm{~h}$. Although obtained using a different technique (involving variations in the brightness of the comet to infer the rotation rate), the Swift data are consistent with the initial changes measured from the cyanogen emission. Seven other comets have previously shown changes in periodicity, but $41 \mathrm{P}$ is an outlier, plus it is rotationally slow, highly surface-active and has a small nucleus. Extrapolating the rotation rates, Bodewits et al. show that the comet may be nearing too slow a rotation speed to maintain gyroscopic stability against outgassing torques, which could then lead to an excited, unstable rotation state of the nucleus, possibly causing the nucleus to spin up again, albeit around a different rotational axis.

May Chiao

Published online: 22 January 2018

https://doi.org/10.1038/s41550-018-0389-0 